\title{
Information for Contributors to Journal of Materials Research
}

1. Articles. Journal of Materials Research publishes original research reports that include complete, detailed, self-contained descriptions of research efforts. All articles must contain an abstract (see item 4).

2. Rapid Communications. Journal of Materials Research also publishes short submissions (i.e., not more than four published pages), which contain important new results and are intended for accelerated publication. Rapid Communications are given priority in handling. An abstract is required.

3. Commentaries and Review. Journal of Materials Research occasionally publishes commentaries on topics of current interest or reviews of the literature in a given area. An abstract is required.

4. Abstract. An abstract must accompany each article. The abstract should be typed (double-spaced) on a separate page., It should be adequate as an index and as a summary. It should give all subjects, major and minor, concerning which new information is presented. It should give the conclusions of the article and all numerical results of general interest. The abstract should avoid the use of jargon and other terminology which would render its content inaccessible to the nonspecialist. The abstract is usually reprinted verbatim in abstract journals.

All abstracts are indexed according to a materials classification scheme and a topical keyword listing. Descriptions of both indexing procedures are available from the editorial office. Authors are expected to provide the editorial office with suitable index classifications for their manuscripts prior to the acceptance of any manuscript for publication.

Authors also may classify their work according to the 1985 version of the Physics and Astronomy Classification Scheme (PACS). A description of this scheme appears in the December, 1984 issue of Physics Today. Although authors' use of this PACS classification is optional, it renders an author's work more readily available in automated retrieval systems, provided the subject matter of the work is appropriate for the PACS scheme. If the authors fail to indicate a PACS classification, the AIP will provide one for them.

5. Manuscripts. Manuscripts are accepted with the understanding that they have not been copyrighted, published, or accepted for publication elsewhere. Manuscripts must be in English, typewritten, double-spaced on one side of the page, on $81 / 2 \times 11 \mathrm{in}$. durable, opaque, white paper. An original and two (2) copies must be submitted. Margins should be a minimum of $1 \mathrm{in}$. on both sides of the paper to permit editorial notations. Authors should use an identical format for their names in all publications to facilitate use of citations and author indexes. Manuscripts must be submitted with a cover letter containing the name, address, and telephone number of the author to whom correspondence should be addressed. No original artwork will be returned without a specific request in writing.

6. Style. Authors are expected to follow the conventional writing, notation, and illustration style prescribed in the American Institute of Physics Style Manual which is available from the AIP, 335 East 45th St., New York, NY 10017 for $\$ 7.50$ prepaid. Authors should also study the form and style of printed material in this journal. The SI units should be used.

7. Illustrations. All illustrations must accompany submitted manuscripts and, like the text, must be in triplicate (one original and two copies). Every illustration must be referred to in the main text in consecutive numerical order. A caption (legend) must accompany each illustration. Captions must be typed double-spaced on a sheet of paper at the end of the manuscript. Drawings should be planned with oversize lettering and widespaced coordinates to be reproduced satisfactorily within single-column width ( $85 \mathrm{~mm}$, or $33 / 8 \mathrm{in}$.). Principal letters on figures are reduced to approximately $1.5 \mathrm{~mm}$ or $1 / 16 \mathrm{in}$. or, if a Leroy lettering device is used, to $\# 060$, regardless of the overall size of the drawing. For best results, draftsmen should be given a recent copy of this or any other journal published by the American Institute of Physics to familiarize themselves with the proportions to be followed.

Line drawings are preferable to photographs and must be made with India ink on plain white paper or tracing cloth. Coordinate paper is not acceptable. Illustrations must be held, with rarest exceptions, to $81 / 2 \times 11 \mathrm{in}$. High-quality glossy prints with sharp even lines and lettering may be submitted in place of original drawings when these are of exaggerated size and awkward to handle.

Photographs of standard apparatus in a laboratory setup are not instructive and should not be included. No heavy writing should be made on the backs of photographs nor should they be clipped or stapled so as to mar the picture. The figure number and the author's name should appear on the back of each illustration. All photographs, including micrographs, should be good quality, continuous tone black-and-white prints. Micrographs should contain a fiducial mark which indicates explicitly the scale length being shown. Specification of the reduction ratio in the caption is not adequate. Any arrows or lettering in the micrograph must satisy the same scaling restrictions as those indicated above for line drawings.

Unless otherwise indicated, all photographs will be reduced to single-column width. Any special instructions regarding the scaling or placement of photographs should be written on the back of all the photographs involved.

Authors must assume responsibility for the quality of computer-generated graphs or charts. It is necessary to exercise control over the computer-driven printer so that the lines of a graph are dark enough for reproduction. If the labeling on a computer-generated plot is of poor quality, professionally hand lettered characters should be superimposed on the plot. Computergenerated plots made up of small horizontal and vertical line segments may be rejected if the segments are not short enough.

8. Mathematics. Mathematical expressions should be type written as completely as possible, with unavailable symbols being carefully inserted in ink. Special care should be given to make equations and formulas clear to the typesetter, and each letter or symbol inserted in the manuscript in handwriting should be identified in the margin the first time it occurs in the text. Capital and lower-case letters should be distinguished clearly where there could be confusion. Fractional exponents should be used to avoid root signs. Extra symbols should be introduced to avoid complicated exponents or where it is necessary to repeat a complicated expression a number of times. The solidus (/) should be used wherever possible for fractions. Mathematical derivations that are easily found elsewhere in the literature should not be used. Rather the references should be cited

9. References. References should be collected and typed (double-spaced) on a separate page. They should be numbered consecutively and arranged thus:

1. A.B. Smith, Phys. Rev. 41, 852 (1932). Continued 
Continued from previous page

2. H. Lamb, Hydrodynamics (Cambridge U.P., Cambridge, England, 1940), 6th ed., pp. 573, 645 .

A list of the abbreviations for the names of journals appears in the Style Manual.

10. Tables. All but the simplest tabular material should be organized into separate tables. Tables should be numbered with Roman numerals and typed on sheets at the end of the manuscript. Captions should be sufficiently descriptive to make the data in the table intelligible without referring to the text. Complicated column headings in the body of the table should be avoided. If necessary, symbols which are explained in the caption should be used. A double horizontal line should be below the caption, a single line below the headings, and another double line at the end of the table. Long tables should, if possible, be submitted in a form ready for direct photoreproduction. Photoreproducible tables (including captions) should be typed single-spaced on an electric typewriter with Elite type or an equivalent proportional-spaced typeface, and should be designed to fit in one journal column $\left(85 \times 250 \mathrm{~mm}\right.$, or $3 \frac{3}{8} \times 97 / 8$ in.) or across two columns $(175 \times 250 \mathrm{~mm}$, or $7 \times 97 / \mathrm{in}$. $)$. Since they will be photoreduced by a factor of 0.75 , photoreduction should be anticipated by making the type-area dimensions of the table $4 / 3$ larger than the final column and page dimensions given above. Detailed instructions for preparing camera-ready tables are available from the Editorial Office.

11. Publication Charges defray a major portion of the cost of the production of this journal. Consequently, authors' institu- tions or companies are urged to pay a publication charge of $\$ 70$ per printed page, which entitles them to 100 reprints. An appropriate form will be mailed to authors upon acceptance of the manuscript.

12. Correspondence. Manuscripts are to be submitted to Dr. Charles B. Duke, Editor in Chief, Journal of Materials Research, Xerox Webster Research Center, 800 Phillips Road, 0114/38D, Webster, NY 14580.

No correspondence should be sent to the Editor about proofs, reprints, or publication charges. Authors will be notified by the Editor if their paper has been accepted for publication. Authors will also be notified when their paper is sent to the American Institute of Physics. All subsequent correspondence about a particular paper should be addressed to the Editorial Supervisor, Journal of Materials Research, American Institute of Physics, 335 East 45th St., New York, NY 10017. Reference must be made to the author, journal, and scheduled date of issue.

13. Proofs of Articles will be sent to the author for review and should be returned promptly to the Editorial Supervisor, Journal of Materials Research. A few alterations in proof are unavoidable, but the cost of making extensive alterations or of correcting mistakes caused by careless preparation of the manuscript will be charged to the author. Unless corrected proofs are returned punctually, publication of the paper will be delayed.

\section{Subscription Order Form Journal of Materials Research}

(Frequency: 6 times annually)

1986 Subscription Rates:*

$\square$ \$250-U.S. Surface $\square$ \$257 - Foreign Surface $\square$ \$265-Europe Optional Air Freight $\square$ \$273 - Asia Optional Air Freight

*Non-profit Educational Libraries: Deduct $\$ 50$ from each subscription ordered by May 30, 1986.

Name

Last

First/M.I.

Institution

Address

street and/or p.o. box

city

state/province

postal code

country

Business telephone( )

Remittance must accompany all orders. Foreign payments must be made by check payable in U.S. dollars drawn on a bank in the United States. Letters of credit will not be accepted.

Send subscriptions to: Marketing Services, American Institute of Physics, 335 East 45th Street, New York, NY 10017 